\title{
Effect of low-level light therapy on diabetic foot ulcers: a near-infrared spectroscopy study
}

\author{
Massimo Salvi \\ Daniele Rimini \\ Filippo Molinari \\ Gianni Bestente \\ Alberto Bruno
}




\title{
Effect of low-level light therapy on diabetic foot ulcers: a near-infrared spectroscopy study
}

\author{
Massimo Salvi, ${ }^{a}$ Daniele Rimini,, ${ }^{a, *}$ Filippo Molinari, ${ }^{a}$ Gianni Bestente, ${ }^{b}$ and Alberto Bruno ${ }^{c}$ \\ aPolitecnico di Torino, Biolab, Department of Electronics and Telecommunications, Turin, Italy \\ bIstituto Superiore Mario Boella (ISMB), Turin, Italy \\ ${ }^{\mathrm{C}} \mathrm{AOU}$ Città della Salute e della Scienza di Torino-San Giovanni Antica Sede, Diabetology Department, Turin, Italy
}

\begin{abstract}
Diabetic foot ulcer (DFU) is a diabetic complication due to peripheral vasculopathy and neuropathy. A promising technology for wound healing in DFU is low-level light therapy (LLLT). Despite several studies showing positive effects of LLLT on DFU, LLLT's physiological effects have not yet been studied. The objective of this study was to investigate vascular and nervous systems modification in DFU after LLLT. Two samples of 45 DFU patients and 11 healthy controls (HCs) were recruited. The total hemoglobin (totHb) concentration change was monitored before and after LLLT by near-infrared spectroscopy and analyzed in time and frequency domains. The spectral power of the totHb changes in the very-low frequency (VLF, $20 \mathrm{Ho} 60 \mathrm{mHz}$ ) and low frequency (LF, 60 to $140 \mathrm{mHz}$ ) bandwidths was calculated. Data analysis revealed a mean increase of totHb concentration after LLLT in DFU patients, but not in HC. VLF/LF ratio decreased significantly after the LLLT period in DFU patients (indicating an increased activity of the autonomic nervous system), but not in $\mathrm{HC}$. Eventually, different treatment intensities in LLLT therapy showed a different response in DFU. Overall, our results demonstrate that LLLT improves blood flow and autonomic nervous system regulation in DFU and the importance of light intensity in therapeutic protocols. () 2017 Society of Photo-Optical Instrumentation Engineers (SPIE) [DOI: 10.1117/1.JBO.22.3.038001]
\end{abstract}

Keywords: low-level light therapy; diabetic foot ulcers; near-infrared spectroscopy.

Paper 160668RR received Sep. 29, 2016; accepted for publication Feb. 22, 2017; published online Mar. 7, 2017.

\section{Introduction}

Peripheral vascular disease and neuropathy are common complications of diabetes mellitus. ${ }^{1}$ Autonomic nerve damage can also interfere with microcirculation, and this may cause a delay in wounds and ulcers healing. Specifically, the lesions of lower limbs, defined as diabetic foot ulcers (DFU), ${ }^{2,3}$ frequently have a very slow healing process. ${ }^{4}$

A number of treatments for DFU have been largely explored, but only prevention and wound care have emerged as the most suitable. ${ }^{5,6}$ Hence, more efficient methods are still required for accelerating the recovery process and avoiding lower limb amputation. A promising treatment in wound healing is the low-level light therapy (LLLT), a system using low-power light to stimulate cellular function and biological activity. ${ }^{7}$ Recent studies showed the importance of LLLT in pain, ${ }^{8,9}$ skin, ${ }^{7,10}$ and wound ${ }^{11,12}$ care. In recent years, investigators have examined the effect of LLLT on the DFU treatment, ${ }^{13}$ finding improvement in pain scores ${ }^{14-16}$ or in clinical foot sensation. ${ }^{17,18}$ However, there is very little scientific understanding of the effective mechanism of LLLT on DFU. Animal studies revealed that LLLT improved skeletal muscle repair, ${ }^{19}$ and vascular and nerve recovery. ${ }^{20}$ Furthermore, an in vitro study observed that LLLT increases oxygen consumption rates, reduces reactive oxygen species concentration, and increases adenosine triphosphate (ATP) production. ${ }^{21}$

However, no previous studies investigated the physiological effect of LLLT on the neuropathy in diabetic patients.
Considering neuropathy affects innervation of the small muscles of the foot and fine vasomotor control of the lower limb circulation, ${ }^{3}$ the effect of LLLT on both the aspects should be examined. Vascular and peripheral nervous systems can be noninvasively explored by near-infrared spectroscopy (NIRS), which quantifies the relative concentration of tissue hemoglobin. ${ }^{22}$ Despite the NIRS general use of monitoring cerebral vascular reactivity, ${ }^{23}$ it can also be used to monitor the diabetic muscle metabolic changes. ${ }^{24-26}$ Furthermore, the analysis of NIRS signal in frequency domain can reveal the nervous system oscillations, and so the progression of peripheral arterial disease and neuropathy can be studied. ${ }^{27,28}$

The aims of this study were twofold: (i) to investigate the effect of LLLT on peripheral vascular and nervous systems in DFU patients and (ii) to test two different LLLT power intensities. A group of diabetic subjects with DFU underwent an LLLT treatment. The modifications in tissue oxygenation and autonomic nervous system regulation were recorded by NIRS. The physiological response of DFU patients to the LLLT protocol was compared with that of healthy subjects.

\section{Materials and Methods}

This study aimed at investigating the effects of LLLT in DFU subjects by NIRS pretreatment and posttreatment monitoring. Furthermore, a healthy control (HC) underwent the same protocol, to compare different effects of LLLT. 
Table 1 Demographic and anamnestic characteristics of the two samples.

\begin{tabular}{lcc}
\hline & Parameter & \\
Group & Value \\
\hline DFU & No. & \\
& Sex $(\mathrm{M} / \mathrm{F})$ & 45 \\
& Age $( \pm \mathrm{SD})$ & $18 / 27$ \\
& Disease duration $(\mathrm{YY} \pm \mathrm{SD})$ & $23.50 \pm 12.13$ \\
$\mathrm{HC}$ & Ulcer duration $(\mathrm{YY} \pm \mathrm{SD})$ & $2.83 \pm 1.53$ \\
& & \\
& No. & 11 \\
& Sex $(\mathrm{M} / \mathrm{F})$ & $5 / 6$ \\
& Age $( \pm S D)$ & $51.26 \pm 5.33$ \\
\hline
\end{tabular}

\subsection{Patients}

A total of 56 subjects were enrolled in this study: 45 patients affected by DFU and 11 HCs. Subjects were recruited in the Diabetology Department, San Giovanni Antica Sede, Torino, Italy. Inclusion criteria for the DFU group were: (1) type 2 diabetes mellitus for at least 1 year and (2) presence of a DFU more than 6 months. Lesions should not be infected (all the lesions were tested by a culture exam) and located in the second half of the lower limb (see below). Exclusion criteria were pregnancy and unstable health conditions (i.e., heart disease). A comparison group of 11 subjects without any history of metabolic, neurological, and vascular diseases were engaged to form the HC group.

The San Giovanni Antica Sede Hospital, where the study was carried out, gave the approval for this study. All of the subjects were informed about the study methods and objectives and signed an informed consent.

The demographic and anamnestic patients' profiles are described in Table 1. There was no significant difference in age group between the DFU and the HC groups.

\subsection{Experimental Protocol}

Our protocol consisted of a single LLLT treatment for each subject. The experiments were carried out in a quiet, dimmed room, at a controlled temperature. Subjects were asked to lie down on an orthopedic bed in a comfortable and supine position, wearing protection goggles. Afterward, the bandages were slightly removed, and wounds were treated with sodium chloride solution $(0.9 \%$ saline solution). A commercially available device (Healite II, Lutronic Corporation, Korea) was used. The light source consisted of 10 arrays of 150 continuous pulse light-emitting diode (LED), with a spot size of $1.75 \mathrm{~cm}$ and the following three wavelengths: 415, 633, and $830 \mathrm{~nm}$. To focus the light beam on the wound, we moved the LED array nearly $10 \mathrm{~cm}$ far away from the wound surface. Then, we adjusted the light head to adapt to the patient's limb shape.

We monitored the local physiological response to LLLT by NIRS before and after treatment. A custom-made frequency-

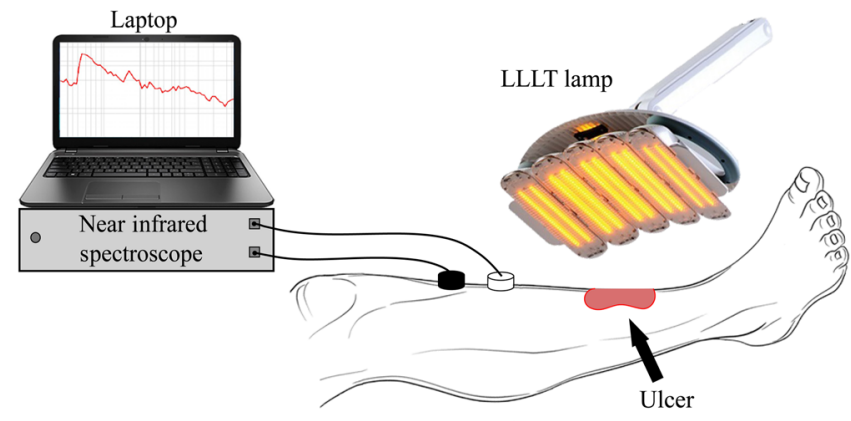

Fig. 1 Position and configuration of the LLLT lamp on the wounded limb. NIRS emitter (white probe) and receiver (black probe) are positioned just above the light head.

resolved single channel NIRS device was adopted, ${ }^{29}$ and modification in concentration of total hemoglobin (totHb) was continuously measured. ${ }^{30}$ The emitter source was formed by three infrared LEDs emitting at 730-, 830-, and 870-nm wavelengths, whereas the receiver was an avalanche photodiode. The receiver probe was located next to the proximal area of tibialis anterior muscle of the wounded leg, whereas the emitter was placed $3 \mathrm{~cm}$ away along the muscle axis. Signals were acquired at a sampling frequency of $10 \mathrm{~Hz}$, low-pass filtered at $1 \mathrm{~Hz}$, then sent to a PC laptop by a USB cable. Figure 1 shows position and configuration of the infrared lamp and NIRS probe during the acquisition. The same setup was used for the HC group (for controls, the leg was randomly selected).

Two configurations of the LLLT device were used. A basic treatment $\left(\mathrm{INT}_{1}\right)$ and a second treatment $\left(\mathrm{INT}_{4}\right)$ with four times the energy of the first treatment were set

1. $\mathrm{INT}_{1}$ : wavelength $=830 \mathrm{~nm}$, energy $=20 \mathrm{~J} / \mathrm{cm}^{2}$, and time $=10.30 \mathrm{~min}$ and

2. $\mathrm{INT}_{4}$ : wavelength $=830 \mathrm{~nm}$, energy $=80 \mathrm{~J} / \mathrm{cm}^{2}$, and time $=17.30 \mathrm{~min}$.

For both configurations, the higher wavelength was chosen, in order to maximize the beam penetration. The 830-nm wavelength was adopted, as target tissues for LLLT are located deeper under the skin. ${ }^{31}$

Both groups (DFU and HC) underwent a single LLLT treatment. Experimental protocol was divided into three observation windows

1. pre-LLLT: NIRS-only signal acquisition before LLLT treatment $(4 \mathrm{~min})$,

2. LLLT therapy, and

3. post-LLLT: NIRS-only signal acquisition after LLLT treatment (4 $\mathrm{min})$.

We were not able to measure physiological modifications during the LLLT therapy (observation window 2) due to the huge amount of photons injected that saturated the NIRS receiver during LED treatment. Figure 2 shows the LLLT protocol and the corresponding NIRS windows.

\subsection{Data Analysis}

Once recorded, NIRS data were downsampled at $2 \mathrm{~Hz}$ and bandpass filtered between 20 and $250 \mathrm{mHz}$ by means of an IIR digital 


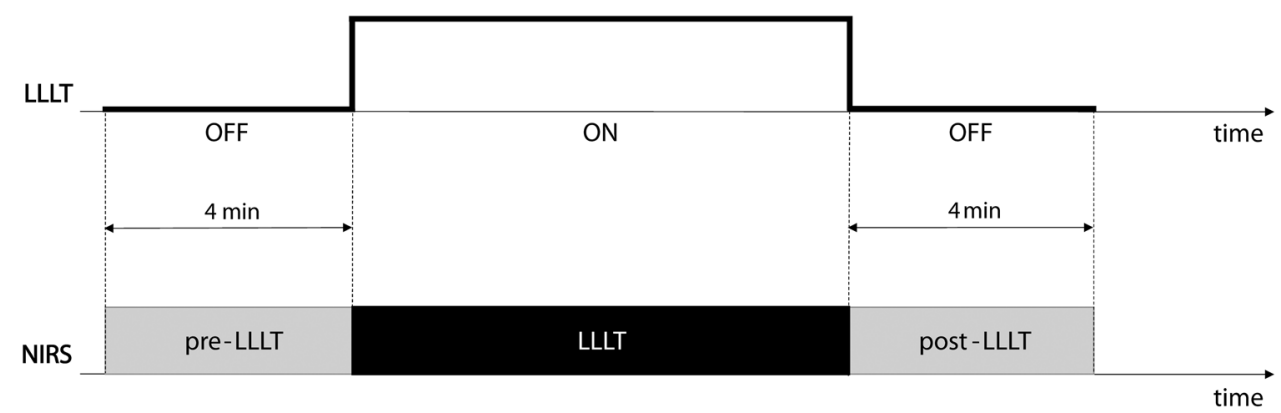

Fig. 2 Acquisition scheme for LLLT and NIRS. LLLT amplitude and duration of ON interval depend on the selected intensity configuration.

(a)

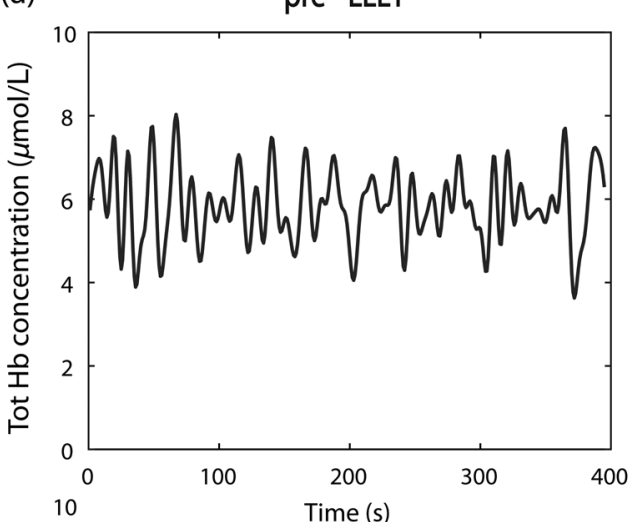

(c)

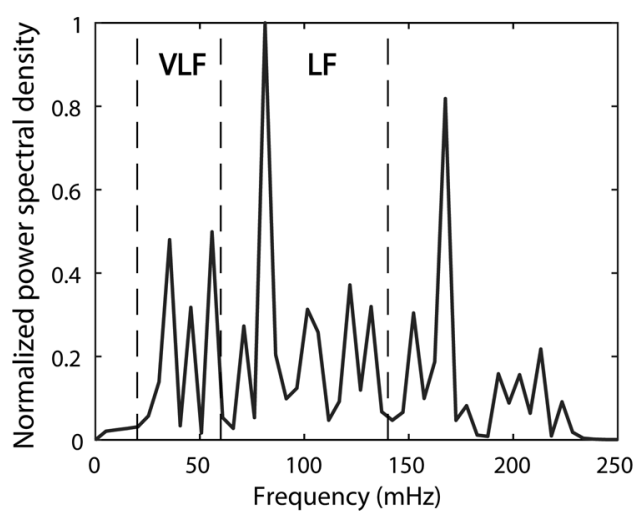

(e)

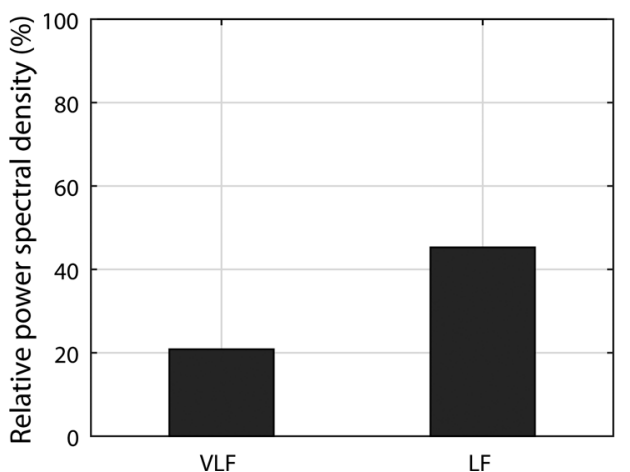

(b)

post - LLLT

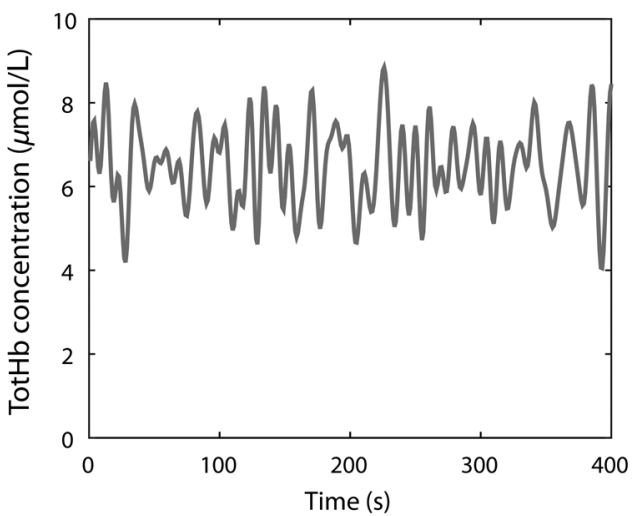

(d)

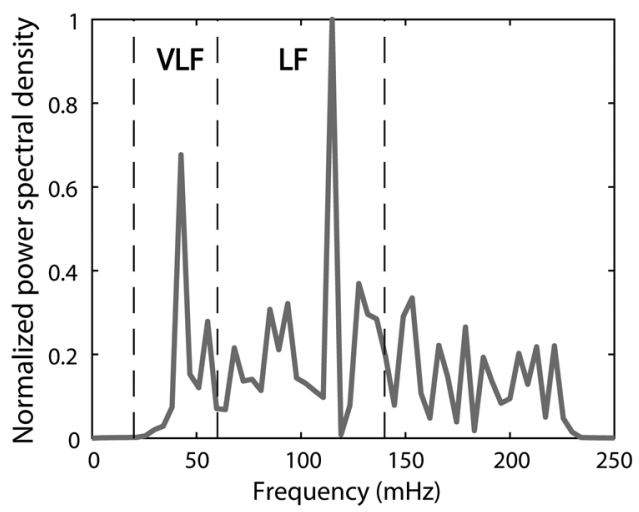

(f)

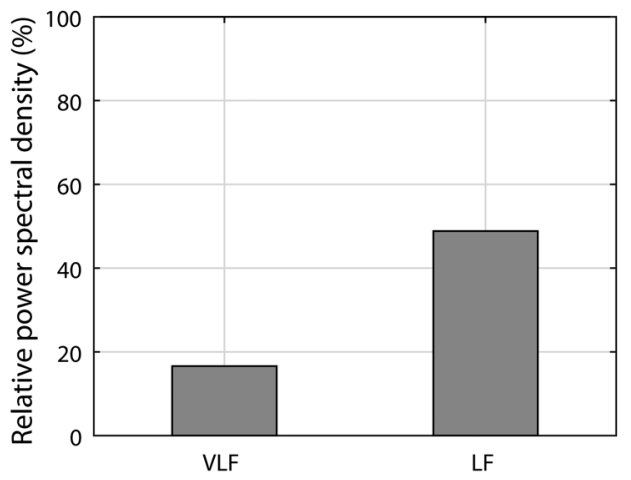

Fig. 3 Example of NIRS data processing for a pre-LLLT and post-LLLT treatment for a representative DFU subject. (a) and (b) Filtered NIRS signals. (c) and (d) Normalized power spectral density estimated from CWs distribution. (e) and (f) Relative power spectral density in VLF and LF bandwidths. 


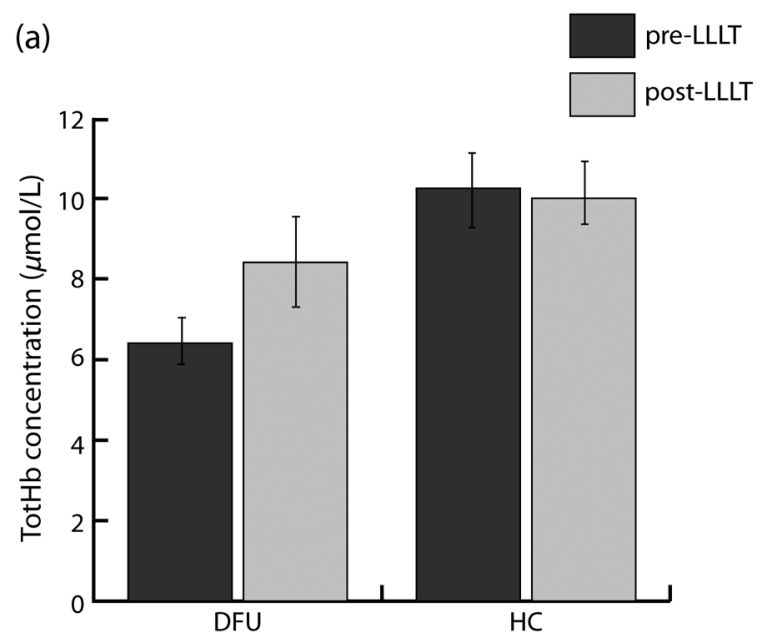

(b)

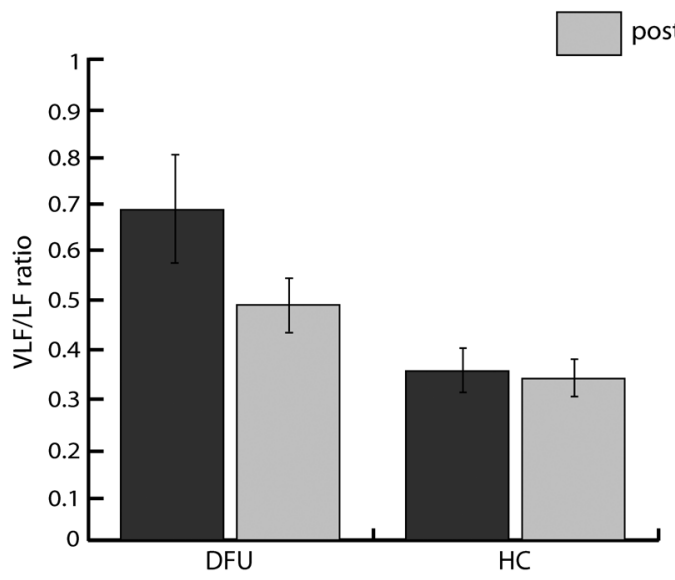

Fig. 4 Average modification in (a) totHb concentration and (b) VLF/LF due to the LLLT in DFU and HC groups. Data are reported as mean \pm standard error of the mean (SEM). A significant increase in totHb and a parallel decrease in VLF/LF can be observed only in the DFU group.

filter (10th-order Chebyshev filter). Filtered NIRS signals were analyzed in time and frequency domains with MATLAB (MathWorks Inc., Massachusetts) custom routines.

In time domain, the mean totHb concentration was compared before (pre-LLLT) and after therapy (post-LLLT) with a Student's paired $t$-test in both DFU and HC groups, to verify different effects of therapy [Figs. 3(a) and 3(b)].

NIRS signals were also analyzed in the frequency domain as slow frequency drifts convey autonomous nervous system information. In NIRS signal, the very-low frequency (VLF) and low frequency (LF) bandwidths are of interest. VLF corresponds to B-waves in the range of 20 to $60 \mathrm{mHz}$ : these oscillations are originated by rhythmic modifications of vessels' diameter. On the other hand, LF correlates to M-waves in the bandwidth of 60 to $140 \mathrm{mHz}$ and represent vascular regulation of the autonomic nervous system. ${ }^{32}$ NIRS signal was analyzed with a time-frequency technique because of its nonstationarity. ${ }^{30,33}$ For this reason, it was analyzed with a time-frequency technique. A time-frequency distribution $D_{x x}(t, f)$ belonging to Cohen class was computed, and a Choi-Williams (CW) distribution was adopted as kernel

$$
\begin{aligned}
D_{x x}(t, f)= & \iiint_{-\infty}^{+\infty} x\left(t^{\prime}-\frac{\tau}{2}\right) x^{*}\left(t^{\prime}+\frac{\tau}{2}\right) g(\tau, \theta) \\
& \cdot e^{j 2 \pi \theta\left(t^{\prime}-t\right)} e^{-j 2 \pi f \tau} \mathrm{d} t^{\prime} \mathrm{d} \theta \mathrm{d} \tau,
\end{aligned}
$$

where

$g(\tau, \theta)=e^{\frac{\theta^{2} \tau^{2}}{\sigma}}$

Scaling factor $\sigma$ is the selectivity of the kernel and may vary from 0.1 to 10 . We chose a value of 0.5 , as it was low enough to attenuate interference terms, but it guaranteed a good representation of frequency components. ${ }^{34,35}$

Once $D_{x x}(t, f)$ was computed for totHb in pre-LLLT and post-LLLT windows for DFU and HC groups, power spectral density was extracted from CW distribution within the bands of interest [Figs. 3(c) and 3(d)]. From the estimated relative power densities, the ratio between VLF and LF power (VLF/ LF) was calculated for pre-LLLT and post-LLLT observation windows [Figs. 3(e) and 3(f)]. Then, they were compared with a paired $t$-test. All statistical tests were carried out with a significance level alpha of 0.05 .

\section{Results}

For each subject, average totHb concentration and VLF/LF ratio were calculated. Then, pre-LLLT versus post-LLLT comparison was carried out in the DFU and control groups. Figure 4 compares modification in mean totHb and VLF/LF ratio in DFU and $\mathrm{HC}$ groups.

As shown in Fig. 4(a), in the diabetic group, mean totHb increased from 6.26 to $8.64 \mu \mathrm{mol} / \mathrm{L}$ after therapy. On the contrary, $\mathrm{HC}$ subjects showed nearly the same totHb concentration. The paired $t$-test revealed a significant difference in totHb concentration for the DFU group $(T=2.49, p=0.017)$, whereas no significant difference was found in the HC group.

Similarly, VLF/LF was compared in DFU and HC groups before and after the therapy. As reported in Fig. 4(b), the VLF/LF ratio was strongly decreased from 0.689 to 0.491 for the DFU group. The decrease in VLF/LF was due to a decrease in VLF and a parallel increase in LF. On average, VLF diminished from $22.8 \%$ to $19.5 \%$, whereas LF elevated from $40.5 \%$ to $43.4 \%$. In contrast, and similar to totHb concentration, VLF/LF in HC during pre-LLLT remained almost equal to the post-LLLT window. A Student's paired $t$-test confirmed a significant difference in VLF/LF in the DFU group $(T=2.17, p=0.035)$, but not in the HC group. The results of the statistical analysis are shown in Table 2.

Table 2 Paired $t$-test of pre-LLLT and post-LLLT totHb and VLF/LF ratio (mean \pm standard error of the mean).

\begin{tabular}{lcccc} 
& & pre-LLLT & post-LLLT & $p$ value \\
\hline DFU & totHb & $6.269 \pm 0.431$ & $8.640 \pm 1.034$ & $0.017^{*}$ \\
& VLF/LF & $0.689 \pm 0.112$ & $0.491 \pm 0.055$ & $0.035^{\star}$ \\
HC & totHb & $10.171 \pm 1.493$ & $10.157 \pm 1.852$ & 0.988 \\
& VLF/LF & $0.353 \pm 0.046$ & $0.337 \pm 0.038$ & 0.835 \\
\hline
\end{tabular}

*indicates a statistically significant difference compared with pre-LLLT 
Of the 56 subjects, $28 \mathrm{DFU}$ patients, and five $\mathrm{HC}$ underwent a lower intensity LLLT $\left(\mathrm{INT}_{1}\right.$ ), whereas $17 \mathrm{DFU}$ and six HC patients were treated at the highest intensity configuration $\left(\mathrm{INT}_{4}\right)$. All subjects have safely concluded the LLLT session and no adverse effects were observed in diseased patients and healthy subjects. The difference between pre-LLLT and postLLLT totHb concentration and VLF/LF ratio were analyzed with the aim to investigate the effect of light energy on body response's magnitude.

In DFU patients, the increment of totHb concentration was higher using the $\mathrm{INT}_{4}$ configuration with respect to the lower intensity setup, whereas in $\mathrm{HC}$, no significant difference was observed [Fig. 5(a)]. There was also a decrease of the VLF/LF ratio in DFU subjects, slightly more at $\mathrm{INT}_{4}$, whereas in $\mathrm{HC}$, two opposite behaviors were noticed using different power light intensities [Fig. 5(b)].

\section{Discussion}

This study aimed at investigating the effect of the light therapy in DFU patients. To this end, we monitored tissue oxygenation during one LLLT treatment using NIRS. Then, we verified the different response to LLLT between diseased patients and healthy subjects. Significant modifications in vascular and nervous systems were observed in DFU patients, but not in HC, whose NIRS parameters after LLLT remained constant. By comparing the NIRS parameters before and after LLLT, we observed a significant increase of totHb concentration and a decrease of $\mathrm{VLF} / \mathrm{LF}$ ratio in DFU patients. The reduction of VLF/LF was due to a lower power in VLF bandwidth and a parallel increase in LF frequencies.

LLLT has been demonstrated to be efficacious in DFU wound treatments. ${ }^{13,36}$ Several hypotheses of DFU physiological response to LLLT have been proposed. Some observed an increase in conduction velocity of the peripheral nerve. ${ }^{18}$ Shashi Kumar et al. ${ }^{16}$ hypothesized an effect of the therapy on peripheral microcirculation due to cytokine and growing

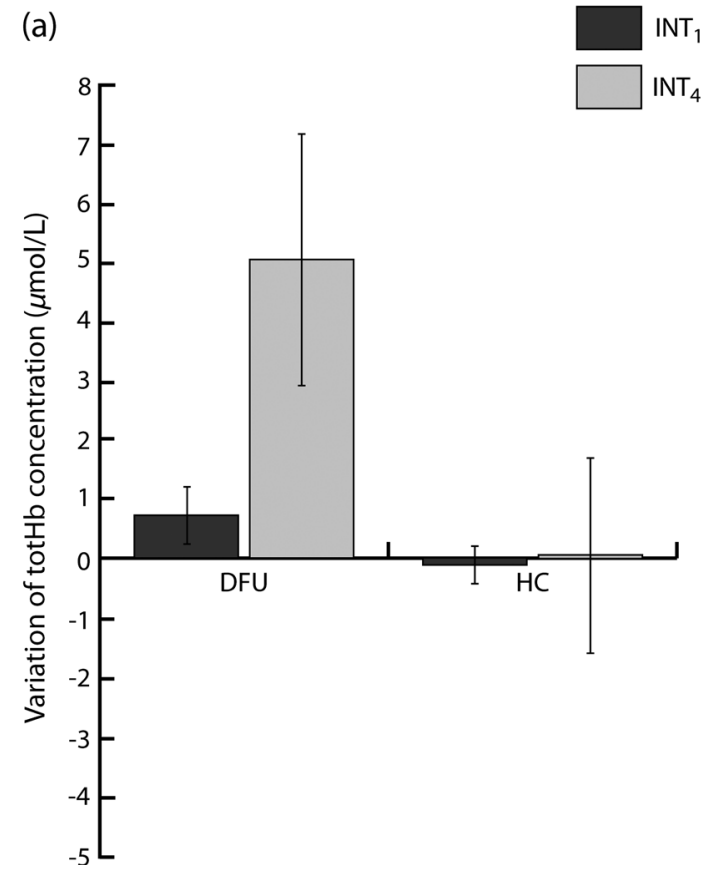

factors release. Recently, alteration of collagen and extracellular matrix synthesis, and migration, proliferation, and differentiation of different types of cells, has been evidenced as possible mechanisms of LLLT action. ${ }^{13}$ In addition, several studies demonstrated the effect of LLLT on increasing the ATP production, ${ }^{10,37,38}$ which may reduce the oxidative stress of the neuropathy. ${ }^{39}$ However, to the best of our knowledge, no study has been performed yet to monitor the physiological parameters during LLLT in diseased patients.

This study showed an increase of totHb concentration in the perilesional area immediately after LLLT. Because totHb is a fingerprint of an increase in the blood volume, ${ }^{30,40}$ its modification may be due to an improvement in blood flow in the wounded region. This increase may be due to the release of growing factors, as previously described. Furthermore, an improvement in the regulation of the vascular tone may contribute to the local vasodilatation as LLLT has been demonstrated to stimulate nitric oxide release. ${ }^{41,42}$ Previous studies proved that LLLT stimulates the regeneration of nervous fibers. ${ }^{43,44}$ On the other hand, modification in slow oscillations of the NIRS signal reflects the regulation of the autonomic nervous system. An increase of power density in LF bandwidth, combined with a drop in the VLF spectrum, may be due to an improvement of autoregulation of the peripheral nervous system in DFU. ${ }^{15,18,21}$

This study has some limitations. First, only one therapeutic session for each patient was performed: this is because we aimed at gathering information about physiological effects of LLLT on peripheral neuropathy and not on the therapeutic efficacy of long-term trials on wound healing. Furthermore, NIRS measures relative concentrations with respect to a zero point. For this reason, multiple sessions cannot be directly compared. By using NIRS, we documented microcirculation and nervous modifications, but information, such as time to response was not measured. Nevertheless, by comparing parameters before and after the therapy, the overall effect of LLLT on vascular and nervous systems was revealed.

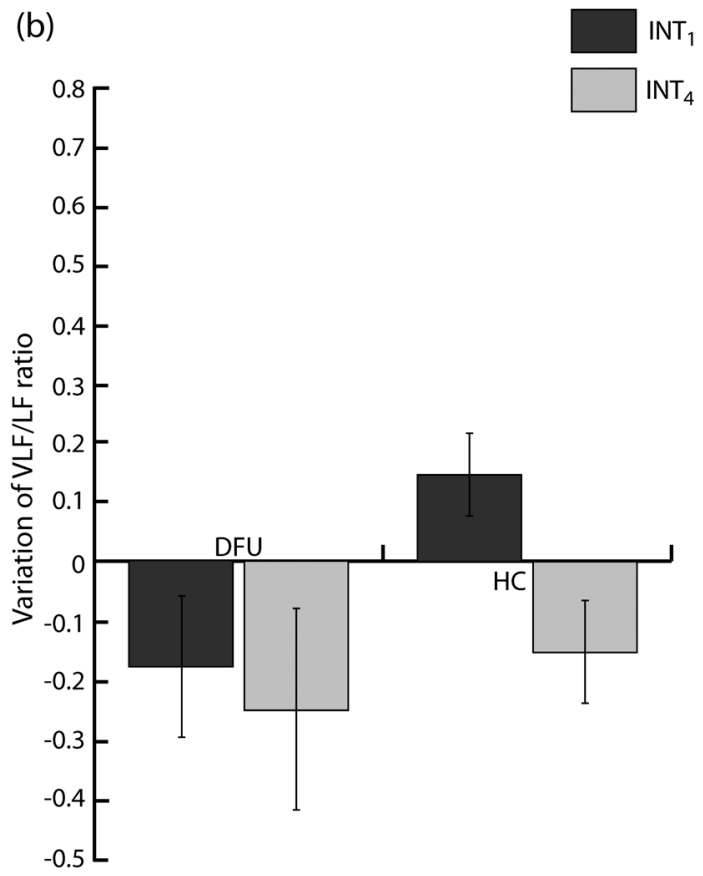

Fig. 5 Effect of LLLT light intensity in (a) totHb concentration and (b) VLF/LF ratio in DFU and HC groups. Data are reported as mean $\pm S E M$. 
The modification of NIRS parameters after a series of treatments and their comparison with quantitative measurements of wound healing will be addressed in future work.

\section{Conclusion}

In this study, we have investigated the changes in nervous system regularization and in tissue perfusion after LLLT on the wounded lower limb of DFU patients and normal subjects. The changes were monitored noninvasively by a NIRS system. We have observed an improvement in both vascular and nervous systems' response in the pathological group because the blood flow increased and the autonomous nervous system regulation improved after the therapy. Furthermore, the variation of totHb concentration was strongly different using two different light intensities in DFU patients. This result highlighted the importance of light intensity in therapeutic protocols, which will be investigated in future studies.

\section{Disclosures}

The authors declare that they have no relevant financial interest in the paper and no other potential conflict of interest.

\section{References}

1. M. Zubair, "Diabetic foot ulcer: a review," Am. J. Intern. Med. 3(2), 28 (2015).

2. W. J. Jeffcoate and K. G. Harding, "Diabetic foot ulcers," Lancet 361(9368), 1545-1551 (2003).

3. B. C. Callaghan et al., "Diabetic neuropathy: clinical manifestations and current treatments," Lancet Neurol. 11(6), 521-534 (2012).

4. C. Chao and G. Cheing, "Microvascular dysfunction in diabetic foot disease and ulceration," Diabetes Metab. Res. Rev 25(7), 604-614 (2009).

5. J. Hwang and R. E. Weiss, "Steroid-induced diabetes: a clinical and molecular approach to understanding and treatment," Diabetes. Metab. Res. Rev 30(2), 96-102 (2014).

6. G. Mulder, M. Tenenhaus, and G. F. D'souza, "Reduction of diabetic foot ulcer healing times through use of advanced treatment modalities," Int. J. Low. Extrem. Wounds 13(4), 335-346 (2014).

7. P. Avci et al., "Low-level laser (light) therapy (LLLT) in skin: stimulating, healing, restoring," Semin. Cutan. Med. Surg. 32(1), 41-52 (2013).

8. R. Yousefi-Nooraie, E. Schonstein, and K. Heidari, "Low level laser therapy for nonspecific low-back pain," Cochrane Database Syst. Rev 2, 2-4 (2007)

9. S. Haslerud et al., "The efficacy of low-level laser therapy for shoulder tendinopathy: a systematic review and meta-analysis of randomized controlled trials," Physiother. Res. Int. 20(2), 108-125 (2015).

10. S. Tsai et al., "Low-level light therapy potentiates NPe6-mediated photodynamic therapy in a human osteosarcoma cell line via increased ATP," Photodiagn. Photodyn. Ther. 12(1), 123-130 (2015).

11. S. Percival, I. Francolini, and G. Donelli, "Low-level laser therapy as an antimicrobial and antibiofilm technology and its relevance to wound healing," Future Microbiol. 10(2), 255-272 (2015).

12. P. K. Min and B. L. Goo, " $830 \mathrm{~nm}$ light-emitting diode low level light therapy (LED-LLLT) enhances wound healing: a preliminary study," Laser Ther. 22(1), 43-49 (2013).

13. C. N. Tchanque-Fossuo et al., "A systematic review of low-level light therapy for treatment of diabetic foot ulcer," Wound Repair Regen. 24(2), 418-426 (2016).

14. L. H. Zinman et al., "Low-intensity laser therapy for painful symptoms of diabetic sensorimotor polyneuropathy: a controlled trial," Diabetes Care 27(4), 921-924 (2004).

15. H. Bashiri, "Evaluation of low level laser therapy in reducing diabetic polyneuropathy related pain and sensorimotor disorders," Acta Med. Iran. 51(8), 543-547 (2013).

16. C. G. Shashi Kumar et al., "Efficacy of low level laser therapy on painful diabetic peripheral neuropathy," Laser Ther. 24(3), 195-200 (2015).
17. L. B. Harkless et al., "Improved foot sensitivity and pain reduction in patients with peripheral neuropathy after treatment with monochromatic infrared photo energy-MIRE," J. Diabetes Complications 20(2), 81-87 (2006).

18. M. E. Khamseh et al., "Diabetic distal symmetric polyneuropathy: effect of low-intensity laser therapy," Lasers Med. Sci. 26(6), 831-835 (2011).

19. M. C. P. Feitosa et al., "Effects of the low-level laser therapy (LLLT) in the process of healing diabetic foot ulcers," Acta Cir. Bras. 30(12), 852-857 (2015).

20. C. M. França et al., "Effect of laser therapy on skeletal muscle repair process in diabetic rats," Lasers Med. Sci. 28(5), 1331-1338 (2013).

21. Y. L. Hsieh et al., "Low-level laser therapy alleviates neuropathic pain and promotes function recovery in rats with chronic constriction injury: possible involvements in hypoxia-inducible factor $1 \alpha(\mathrm{HIF}-1 \alpha)$," J. Comp. Neurol. 520(13), 2903-2916 (2012).

22. R. Boushel et al., "Monitoring tissue oxygen availability with near infrared spectroscopy (NIRS) in health and disease," Scand. J. Med. Sci. Sports 11(4), 213-222 (2001).

23. R. Spitler et al., "Combination of low level light therapy and nitrosylcobinamide accelerates wound healing," J. Biomed. Opt. 20(5), 051022 (2015).

24. F. Molinari et al., "Empirical mode decomposition analysis of nearinfrared spectroscopy muscular signals to assess the effect of physical activity in type 2 diabetic patients," Comput. Biol. Med. 59, 1-9 (2015).

25. F. Molinari et al., "Entropy analysis of muscular near-infrared spectroscopy (NIRS) signals during exercise programme of type 2 diabetic patients: quantitative assessment of muscle metabolic pattern," Comput. Methods Programs Biomed. 112(3), 518-528 (2013).

26. M. Ferrari, T. Binzoni, and V. Quaresima, "Oxidative metabolism in muscle," Philos. Trans. R. Soc. Lond. B. Biol. Sci. 352(1354), 677-683 (1997).

27. H. W. Schytz et al., "Spontaneous low-frequency oscillations in cerebral vessels: applications in carotid artery disease and ischemic stroke," J. Stroke Cerebrovasc. Dis. 19(6), 465-474 (2010).

28. S. Rosati, G. Balestra, and F. Molinari, "Feature selection applied to the time-frequency representation of muscle near-infrared spectroscopy (NIRS) signals: characterization of diabetic oxygenation patterns," J. Mech. Med. Biol. 12(04),1240013 (2012).

29. G. Themelis et al., "Depth of arterial oscillation resolved with NIRS time and frequency domain," in Biomedical Topics Meeting WF2 (2004).

30. M. Ferrari and V. Quaresima, "A brief review on the history of human functional near-infrared spectroscopy (fNIRS) development and fields of application," Neuroimage 63(2), 921-935 (2012).

31. R. B. Panerai, "Complexity of the human cerebral circulation," Philos. Trans. A. Math. Phys. Eng. Sci. 367(1892), 1319-1336 (2009).

32. U. Sliwka et al., "Spontaneous oscillations in cerebral blood flow velocity give evidence of different autonomic dysfunctions in various types of headache," Headache 41(2), 157-163 (2001).

33. F. Molinari et al., "Ozone autohemotherapy induces long-term cerebral metabolic changes in multiple sclerosis patients," Int. J. Immunopathol. Pharmacol. 27(3), 379-389 (2014).

34. H. I. Choi and W. J. Williams, "Improved time-frequency representation of multicomponent signals using exponential kernels," IEEE Trans. Acoust. 37(6), 862-871 (1989).

35. P. Castiglioni, "Choi-Williams distribution," in Encyclopedia of Biostatistics, John Wiley \& Sons, Ltd., Chichester (2005).

36. H. T. Whelan et al., "Effect of NASA light-emitting diode irradiation on wound healing," J. Clin. Laser Med. Surg. 19(6), 305-314 (2001).

37. J. Tafur et al., "Biophoton detection and low-intensity light therapy: a potential clinical partnership," Photomed. Laser Surg. 28(1), 23-30 (2010).

38. C. Ferraresi et al., "Time response of increases in ATP and muscle resistance to fatigue after low-level laser (light) therapy (LLLT) in mice," Lasers Med. Sci. 30(4), 1259-1267 (2015).

39. T. Zhang, Y. Gong, and H. Zhou, "Oxidative stress-the key mechanism of diabetic peripheral neuropathy," North Am. J. Med. Sci. 6(2), 87-90 (2013).

40. I. Tachtsidis et al., "Quantification of adult cerebral blood volume using the NIRS tissue oxygenation index," Adv. Exp. Med. Biol. 578, 237-243 (2006). 
41. P. Dungel et al., "Low level light therapy by LED of different wavelength induces angiogenesis and improves ischemic wound healing," Lasers Surg. Med. 46(10), 773-780 (2014).

42. M. A. Takhtfooladi et al., "Effect of low-level laser therapy on lung injury induced by hindlimb ischemia/reperfusion in rats," Lasers Med. Sci. 30(6), 1757-1762 (2015).

43. W. Xuan et al., "Low-level laser therapy for traumatic brain injury in mice increases brain derived neurotrophic factor (BDNF) and synaptogenesis," J. Biophotonics 8(6), 502-511 (2015).

44. R. L. Buchaim et al., "Effect of low-level laser therapy (LLLT) on peripheral nerve regeneration using fibrin glue derived from snake venom," Injury 46(4), 655-660 (2015).

Filippo Molinari is an associate professor on the faculty of the Department of Electronics, Politecnico di Torino. His research topics include the analysis of biosignals and the biomedical image processing applied to the computer-aided diagnosis and therapy. He is a member of the IEEE Engineering in Medicine and Biology Society, the Italian Group of Bioengineering, and the American Institute for Ultrasound in Medicine.

Alberto Bruno received his MS degree in diabetology and internal medicine from the University of Turin in 1981 and 1989, respectively. $\mathrm{He}$ is coordinator of the Unified Center of Diabetology and director of the Management of Diabetes Chronic Complications Unit at the San Giovanni Battista Hospital of Turin. He is also the national supervisor of the Italian Society of Diabetology working group on diabetic foot.

Biographies for the other authors are not available. 